\title{
The Complete Primary Structure of the Marine Carnivora, Galapagoes Fur Seal (Arctocephalus galapagoensis, Otariidae) Hemoglobins
}

\author{
Meeno Jahan, ${ }^{1,2,4}$ Aftab Ahmed, ${ }^{1,2}$ Fritz Trillmich, ${ }^{3}$ and Gerhard Braunitzer ${ }^{1,5}$
}

Received January 4, 1991

The complete primary structure of the two hemoglobin components of the fur seal (Arctocephalus galapagoensis) is presented. The two components ( $\mathrm{HbI}$ and $\mathrm{HbII}$ ) occur in nearly equal amounts and have identical $\beta$-chains; whereas the two $\alpha$-chains $(\alpha \mathrm{I} / \alpha \mathrm{II})$ differ by six exchanges Ile/Val, Met/Thr, Ser/Ala, Pro/His, Lys/Gly, and Thr/Ala at positions 10, 34, 35, 50, 78, and 131, respectively. The components were isolated by DEAE-Sephacel chromatography and were separated into the globin chains by RP-HPLC on a column of Nucleocil-C4. The sequences have been determined by Edman degradation in liquid- and gas-phase sequencer, using the native chains and tryptic peptides. The sequences compared with those of other Carnivora species and an adult human globin chains. An identical $\beta$-chain is found in fur seal and walrus, whereas larger differences were found between $\alpha \mathrm{I}$ and $\alpha \mathrm{II}$ compared to $\beta$-chains.

KEY WORDS: Fur seal; marine carnivora; hemoglobin; primary structure; homology.

\section{INTRODUCTION}

Otariid pinnipeds (eared seals) could spend most of their time in the water. For arctic and antarctic pinnipeds, reproduction is one of the main reasons for coming ashore because the young are unable to survive in the water for some time after birth; however, it is much less pronounced in galapagos species, in which even females come all year to inhabitual colonies without new born. Very little information is available on hemoglobin structure and function of pinniped seals. In the superfamily Otarioidea, amino acid sequences of the hemoglobin are so far known only for Pacific walrus (Odobenus rosmarus divergens) hemoglobin, which belongs to family Odobenidae (Lin et

\footnotetext{
'Max-Planck-Institut für Biochemie, Abt. Proteinchemie D-8033, Martinsried bie Müchen, Germany.

${ }^{2}$ Present address: Laboratory of Molecular Virology and Carcinogenesis, National Cancer Institute, P.O. Box B, Frederick, Maryland 21702.

${ }^{3}$ Max-Planck-Institut für Verhaltensphysiologie, Abt. Wickler D-8131, Seewiessen, Germany.

${ }^{4}$ To whom correspondence should be addressed.

${ }^{5}$ Deceased on May 27, 1989.
}

al., 1989). In order to understand the structure-function relationship and evolution at the molecular level, this communication presents the complete primary structure of the $\alpha \mathrm{I}, \alpha \mathrm{II}$, and $\beta$-chains of the fur seal, Arctocephalus galapagoensis, which belongs to the family Otariidae.

\section{MATERIALS AND METHODS}

\subsection{Isolation of Hemoglobin and Electrophoresis}

Blood from fur seal (Arctocephalus galapagoensis) was collected at Galapagos Island in heparinized tubes. The erythrocytes were washed three times with physiological saline and lysed with cold distilled water. The hemolysate was examined for hemoglobin multiplicity by polyacrylamide gel disk electrophoresis in Tris/glycine buffer at $p \mathrm{H} 8.5$ (Davis, 1964). Electrophoresis under dissociating conditions was carried out in the presence of Triton $\mathrm{X}-100$ and $8 \mathrm{M}$ urea (Alter et al., 1980).

\subsection{Separation of Hemoglobin Components}

Whole hemolysate was subjected to ion exchange chromatography on a column $(1.6 \times 16 \mathrm{~cm})$ of 
DEAE-Sephacel (Pharmacia), equilibrated with $50 \mathrm{mM}$ Tris $/ \mathrm{HCl}$ buffer containing $0.01 \% \mathrm{KCN} p \mathrm{H}$ 8.5. Elution of $\mathrm{Hb}$ was carried out with a gradient of $0-0.1 \mathrm{M} \mathrm{NaCl}$. Flow rate was maintained at $20 \mathrm{ml} /$ hr. The absorbance was monitored at 415 and $280 \mathrm{~nm}$.

\subsection{Separation of Globin Chains}

\subsubsection{Ion-Exchange Method}

Hemoglobin was dehemed in cold acetone solution containing 2\% $\mathrm{HCl}$ (Anson et al., 1930). Globin was reduced under nitrogen for $3 \mathrm{~h}$. The $\alpha$ - and $\beta$ chains were separated on a carboxymethylcellulose CM-52 (Whatman) column $(1.6 \times 15.7 \mathrm{~cm})$, equilibrated with $25 \mathrm{mM}$ sodium acetate, $0.2 \%$ mercaptoethanol, and $8 \mathrm{M}$ urea, using a $0.02-0.08 \mathrm{M} \mathrm{NaCl}$ gradient (Clegg et al., 1966).

\subsubsection{RP-HPLC Method}

Globin was prepared from $\mathrm{CN}$-met-hemoglobin components by the acetone- $\mathrm{HCl}$ procedure (Anson et al., 1930), and globin chains were separated by RPHPLC using a 342-Gradient Liquid Chromatography, Controller 421 (Beckman) and a column of Nucleocil-C4 (Macherey \& Nagel). The column was equilibrated with $0.1 \%$ aqueous TFA. Globin chains were eluted with a linear gradient of $35-60 \%$ acetonitrile over $60 \mathrm{~min}$ at a flow rate $1 \mathrm{ml} / \mathrm{min}$. Absorbance was recorded at $230 \mathrm{~nm}$.

\subsection{Enzymatic Cleavage and Fractionation of Peptides}

The globin chains were oxidized with performic acid and digested with trypsin (Tos-PheCH2Cl-

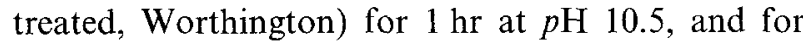
another $2 \mathrm{hr}$ at $p \mathrm{H} 9.5$ with an enzyme/substrate ratio of 5:100 (Hirs et al., 1967). After $3 \mathrm{hr}$, the hydrolysate was titrated to $p \mathrm{H} 4$ and centrifuged. The soluble fraction was subjected to gel filtration on a column of Sephadex G-25 fine $(2.6 \times 140 \mathrm{~cm})$, equilibrated, and eluted with $0.1 \mathrm{M}$ acetic acid at a flow rate of $20 \mathrm{ml} /$ hr. Individual peaks were rechromatographed by RPHPLC on Lichrosorb RP-2 column $(4.6 \times 25 \mathrm{~mm})$, using an ammonium acetate/acetonitrile system (Kratzin et al., 1980). Peptides were eluted with a gradient from $0-60 \%$ over a $60 \mathrm{~min}$, at a flow rate $1 \mathrm{ml} / \mathrm{min}$.

\subsection{Sequence Analysis ${ }^{6}$}

Sequence studies were carried out by automatic Edman degradation (Edman and Begg, 1967) in liquid-phase sequenator (models $890 \mathrm{~B}$ and $890 \mathrm{C}$ Beckman Instruments). For intact polypeptides and lysine peptides, $0.25 \mathrm{M}$ Quadrol was employed (Braunitzer et al., 1970) after coupling with reagent IV (Pfletschinger and Braunitzer, 1980); whereas 3diethylamino propyne was used in the case of arginine peptides (Braunitzer et al., 1978). Some peptides were sequenced by the gas-phase method using the noncommercial sequenator (Begg et al., 1986). Conversion of the thiazolinone to phenylthiohydantoin derivatives of amino acids was carried out in the presence of $3 \mathrm{M} \mathrm{TFA}$ at $80^{\circ} \mathrm{C}$, and identified by HPLC (Zimmermann and Pisano, 1977).

\section{RESULTS AND DISCUSSION}

\subsection{Hemoglobin Composition and Primary Structure}

Alkaline polyacrylamide gel electrophoresis revealed two hemoglobin components occuring in nearly equal amounts (Fig. 1a). Electrophoresis under dissociating conditions (Fig. lb) moreover showed the presence of one $\alpha$ - and one $\beta$-chain. During chain

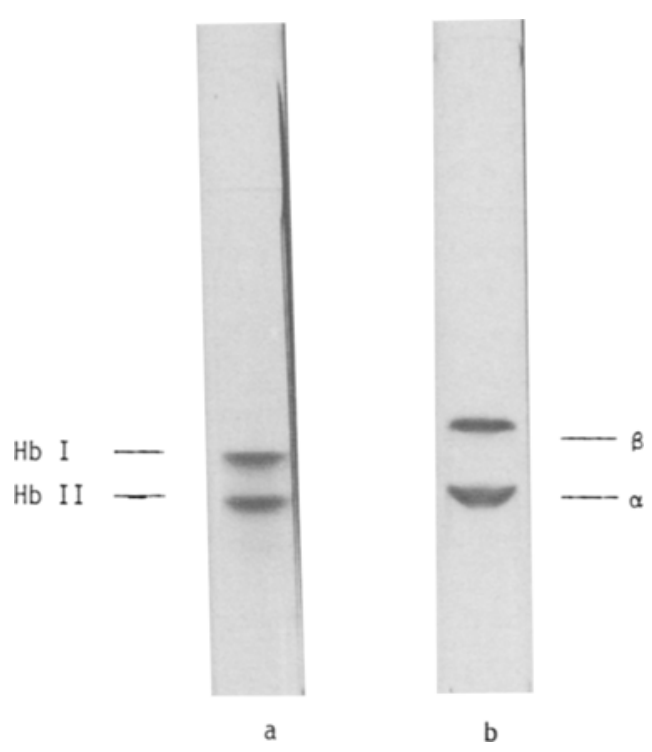

Fig. 1. Electrophoretic pattern of fur seal hemolysate on polyacrylamide gel. (a) Disk electrophoresis at $p \mathrm{H} 8.5$; (b) under dissociating conditions in $8 \mathrm{M}$ urea and Triton X-100.

\footnotetext{
${ }^{6}$ Quadrol = N,N,N,N-tetrakis(2-hydroxypropyl)ethylenediamine; Reagent IV = trisodium 7-(Isothiocyanato)-naphthalene-1,3,5-trisulphonate; Propyne $=3$-(diethylamino) propyne.
} 


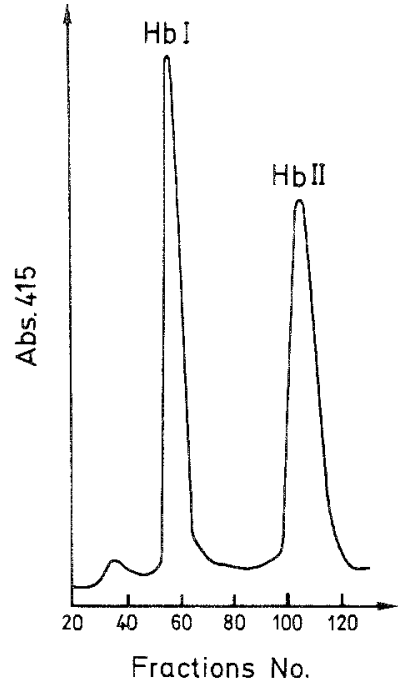

Fig. 2. Separation profile of the hemoglobin components on DEAE-Sephacel $(1.6 \times 16 \mathrm{~cm}), 50 \mathrm{mM}$ Tris-HCl, $p \mathrm{H} 8.5, \mathrm{NaCl}$ gradient $0-0.1 \mathrm{M}$.

separation by CM-cellulose chromatography two chains were eluted. Considering the results of disk electrophoresis and amino acids analysis of the tryptic peptides, which evidence two hemoglobin components, this suggests that the two components differ in the $\alpha$-chains only. Subjecting the native $\alpha$-chain to Edman degradation proves the presence of two $\alpha$-chains with different in charges, explaining the presence of two bands in the alkaline polyacrylamide gel electrophoresis (Fig. 1a).

To obtain homogenous $\alpha \mathrm{I}$ - and $\alpha \mathrm{II}$-chains, the individual components that were separated by DEAESephacel chromatography (Fig. 2) were subjected to RP-HPLC, on Nucleosil column, as shown in Fig. 3. The amino acid compositions of the tryptic peptides obtained from the oxidized chains are listed in Tables I-III. The primary structure of the globin chains was

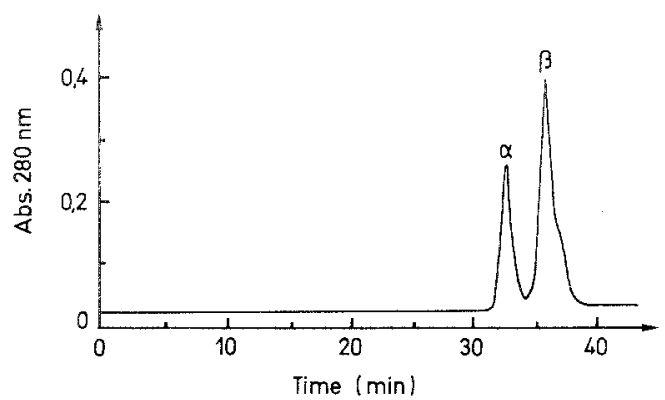

Fig. 3. Chain separation of globin from $\mathrm{Hb}$ II on a RP-HPLC. column : Nucleosil-C4 $(4.6 \times 250 \mathrm{~nm})$; buffer : $0.1 \%$ TFA; gradient : $35-60 \%$ acetonitrile over $60 \mathrm{~min}$; flow rate: $1 \mathrm{ml} / \mathrm{min}$. mainly determined by sequencing the $\mathrm{N}$-terminal regions of the native chains (up to 63 residues of the $\alpha$-chains and 42 of the $\beta$-chain), followed by sequencing of the tryptic peptides. The sequence analysis showed identical primary structures for the $\beta$-chains of both components. The complete primary structures of the $\alpha \mathbf{I}-, \alpha \mathrm{II}-$, and $\beta$-chains are given in Fig. 4. Interestingly, the structural differences in the two hemoglobin components were confined six positions in (four different tryptic peptides) to the $\alpha$-chains, but were resolved neither by electrophoresis under dissociating conditions nor by any other chromatographic methods (CM-cellulose or RP-HPLC). The $\alpha$-II differs from $\alpha$-I at positions 10 (lle/Val), 34 (Met/ Thr), 35 (Ser/Ala), 50 (Pro/His), 78 (Lys/Gly), and 131 (Thr/Ala).

\subsection{Structural and Evolutionary Aspects}

A comparison of $\alpha \mathrm{I}$ - and $\alpha$ II-chains from the fur seal and human hemoglobins indicates $20(14.2 \%)$ and $19(13.5 \%)$ amino acids exchanges, respectively. In the $\beta$-chains, the number of subsitutions is lower: 11 $(7.5 \%)$ replacements distributed over the entire length of the $\beta$-chains. Some of the amino acid residues exchanged in the fur seal are involved in contacts with the heme or between the subunits in human hemoglobin. The heme contact affected is $\beta 70$ (E14) Ala/Ser. $\beta 43$ (CD2) Glu/Asp is an $\alpha 1 \beta 2$ contact, whereas $\beta 125$ (H3) Pro/Gln, $\alpha$ I 34(B15) Leu/Met, $\alpha$ II 34(B15) Leu/Thr, $\alpha$ II35 (B16) Ser/Ala, $\alpha 111$ (G18) Ala/Cys are $\alpha 1 \beta 1$ contacts. Most of these subsitutions are common in other mammals. None of the residues contributing to the 2,3-diphosphoglycerate contact sites was altered. The evolutionary relationships of marine mammals are not fully understood and there are some controversy about the origins of Pinnipedia, that are specialized for an aquatic existence, whether Otarioidea and Ursidae have common ancestors as well as Phocoidea and Mustalidea. More hemoglobin sequences in this suborder may help to clarify origins and evolutionary relationships at the molecular level.

In Tables IV and V, sequences for 12 carnivore $\alpha$ - and $\beta$-chains are compared to fur seal $\alpha \mathbf{I}-, \alpha \mathrm{II}-$, and $\beta$-chains. The Carnivora species are: harbor seal (Watanabe et al., 1986); Weddell seal, pacific walrus (Lin et al., 1989a,b); polecat (Ahmed et al., 1989), European mink (Ahmed et al., 1990); racoon (Brimhall et al., 1978); badger (Hombrados et al., 1976, 1978); lesser panda, giant panda (Tagle et al., 1986); polar bear (Hofman et al., 1987; dog (Brimhall et al., 1977a); and coyote (Runkel et al., 1974). The 
Table I. Amino Acid Composition of Tryptic Peptides of $\alpha$ I-Chain of Fur Seal Hemoglobin

\begin{tabular}{cccccccccccccccc}
\hline Tp & 1 & 2 & 3 & 4 & 5 & 6 & 7 & 8 & $9 \mathrm{a}$ & $9 \mathrm{~b}$ & 10 & 11 & 12 & 13 & 14 \\
\hline Pos. & $1-7$ & $8-11$ & $12-16$ & $17-31$ & $32-40$ & $41-56$ & $57-60$ & 61 & $62-78$ & $79-90$ & $91-92$ & $93-99$ & $100-127$ & $128-139$ & $140-141$ \\
\hline Asp & 1.07 & 1.02 & 0.86 & - & - & 1.06 & - & - & $2.79(3)$ & 1.14 & - & 1.99 & 1.11 & - & - \\
Thr & - & 1.04 & 2.12 & - & 3.10 & 1.01 & - & - & 2.13 & - & - & - & 1.94 & 2.92 & - \\
Ser & 0.82 & - & - & - & 1.28 & $1.78(2)$ & - & - & - & $1.78(2)$ & - & - & $1.78(2)$ & $2.78(3)$ & - \\
Glx & - & - & - & 3.07 & - & 1.04 & - & - & - & - & - & - & 1.07 & - & - \\
Pro & 1.13 & - & - & - & 0.67 & 1.98 & - & - & 0.94 & - & - & 1.19 & 2.22 & - & - \\
Gly & - & - & - & 4.88 & - & 1.08 & 0.95 & - & - & - & - & - & - & - & - \\
Ala & 0.99 & - & - & 2.08 & - & 1.03 & 1.01 & - & 4.15 & 3.04 & - & - & 4.08 & - & - \\
Cys & - & - & - & - & - & - & - & - & - & - & - & - & 1.84 & - & - \\
Val & 0.98 & - & - & - & - & 1.04 & - & - & 2.14 & - & - & 1.85 & 2.10 & 2.07 & - \\
Met & - & - & - & - & 0.96 & - & - & - & 0.95 & - & - & - & - & - & - \\
Ile & - & 1.07 & - & - & - & - & - & - & - & - & - & - & - & - \\
Leu & 1.03 & - & - & 1.98 & - & 1.00 & - & - & 2.00 & 3.00 & 1.00 & - & 5.92 & 1.10 \\
Tyr & - & - & - & 0.90 & - & $0.66(1)$ & - & - & - & 0.90 & - & - & - & - & 0.91 \\
Phe & - & - & - & - & 2.12 & 2.02 & - & - & - & - & - & 0.94 & 0.99 & 2.03 & - \\
His & - & - & - & 1.07 & - & 1.19 & 0.97 & - & 1.00 & 1.10 & - & - & 3.97 & - & - \\
Trp ${ }^{a}$ & - & - & 1.00 & - & - & - & - & - & - & - & - & - & - & - \\
Lys & 0.97 & 1.10 & 1.01 & - & 1.18 & 1.08 & 1.06 & 1.00 & 1.02 & 1.02 & - & 1.06 & 1.02 & 1.10 & - \\
Arg & - & - & - & 1.00 & - & - & - & - & - & - & 0.99 & - & - & - \\
Sum & 7 & 4 & 5 & 15 & 9 & 16 & 4 & 1 & 17 & 12 & 2 & 7 & 28 & 1.01 \\
\hline
\end{tabular}

${ }^{a}$ Determined after performic acid oxidation. Values within parentheses are taken from sequence analysis.

Table II. Tryptic Peptides from $\alpha$ II-Chain of Fur Seal Hemoglobin

\begin{tabular}{ccccccccccccccc}
\hline Tp & 1 & 2 & 3 & 4 & 5 & 6 & 7 & 8 & 9 & 10 & 11 & 12 & 13 & 14 \\
\hline Pos. & $1-7$ & $8-11$ & $12-16$ & $17-31$ & $32-40$ & $41-56$ & $57-60$ & 61 & $62-90$ & $91-92$ & $93-99$ & $100-127$ & $128-139$ & $140-141$ \\
\hline Asx & 1.08 & 1.02 & 0.89 & - & - & 1.02 & - & - & 3.83 & - & 1.98 & 1.00 & - & - \\
Thr & - & 1.04 & 2.11 & - & 3.97 & 0.91 & - & - & 1.94 & - & - & 1.92 & 2.03 & - \\
Ser & 0.80 & - & - & - & - & $1.68(2)$ & - & - & $1.79(2)$ & - & - & 1.84 & 2.79 & - \\
Glx & - & - & - & 3.02 & - & 1.12 & - & - & - & - & - & 1.03 & - & - \\
Pro & 1.13 & - & - & - & 1.12 & 1.18 & - & - & 1.29 & - & 1.09 & - & - & - \\
Gly & - & - & - & 4.89 & - & 1.16 & 0.98 & - & 1.08 & - & - & 2.11 & - & - \\
Ala & 0.99 & - & - & 2.09 & 0.99 & 0.98 & 1.00 & - & 6.82 & - & - & 4.06 & 1.05 & - \\
Cys & - & - & - & - & - & - & - & - & - & - & - & 2.01 & - & - \\
Val & 0.98 & 0.98 & - & - & - & 0.91 & - & - & 1.97 & - & 1.85 & 2.11 & 2.06 & - \\
Met & - & - & - & - & - & - & - & - & 0.92 & - & - & - & - & - \\
Leu & 1.01 & - & - & 1.99 & - & 0.99 & - & - & 5.23 & 1.11 & - & 5.92 & 1.03 & - \\
Tyr & - & - & - & 0.89 & - & 0.85 & - & - & 1.00 & - & - & - & - & 1.00 \\
Phe & - & - & - & - & 1.89 & 1.89 & - & - & - & - & 0.98 & 0.99 & 2.09 & - \\
His & - & - & - & 1.06 & - & 2.19 & 0.89 & - & 2.02 & - & - & 3.91 & - & - \\
Trp & - & - & 1.00 & - & - & - & - & - & - & - & - & - & - & - \\
Lys & 0.98 & 1.05 & 1.11 & - & 1.03 & 1.08 & 1.02 & 1.00 & 1.09 & - & 1.02 & 1.01 & 1.11 \\
Arg & - & - & - & 1.00 & - & - & - & - & - & 1.01 & - & - & - & 1.01 \\
Sum & 7 & 4 & 5 & 15 & 9 & 16 & 4 & 1 & 29 & 2 & 7 & 28 & 12 & 2 \\
\hline
\end{tabular}

${ }^{a}$ Determined after performic acid oxidation. Values within parentheses are taken from sequence analysis. 
Table III. Amino Acid Composition of Tryptic Peptides of $\beta$-Chain of Fur Seal Hemoglobin

\begin{tabular}{|c|c|c|c|c|c|c|c|c|c|c|c|c|c|c|c|c|c|}
\hline$T p$ & 1 & 2 & 3 & 4 & 5 & 6 & 7 & 8 & $9 \mathrm{a}$ & $9 b$ & $10 \mathrm{a}$ & $10 \mathrm{~b}$ & 11 & 12 & 13 & 14 & 15 \\
\hline Pos. & $1-8$ & $9-17$ & $18-30$ & $31-40$ & $41-59$ & $60-61$ & $62-65$ & 66 & $67-76$ & $77-82$ & $83-87$ & $88-95$ & $96-1041$ & $05-1201$ & $21-132$ & 133-144 & $145-146$ \\
\hline Asp & 1.04 & - & 2.17 & - & $4.88^{a}$ & - & - & - & 2.16 & 2.88 & 一 & 1.02 & 2.12 & 1.25 & - & 1.11 & $\ldots$ \\
\hline Thr & 0.81 & 1.03 & - & 1.06 & - & - & - & - & - & - & 1.00 & - & - & - & 0.93 & - & - \\
\hline Ser & 一 & - & - & - & 2.48 & - & - & - & $1.68(2)$ & - & $-\cdots$ & $0.79(1)$ & ) - & - & - & - & - \\
\hline Glu & 1.10 & - & 2.23 & 1.07 & - & - & - & - & - & - & - & 1.02 & 1.08 & - & 3.94 & -- & 一 \\
\hline Pro & - & 一 & 一 & 1.14 & 2.32 & - & - & - & - & - & - & - & 0.90 & - & 1.27 & - & - \\
\hline Gly & - & 1.26 & 2.88 & - & 2.03 & - & 1.09 & - & 1.08 & - & 0.97 & - & - & 1.94 & - & 1.12 & - \\
\hline Ala & 0.93 & 2.88 & 0.94 & - & 1.16 & - & 1.00 & - & - & - & 0.98 & - & - & 1.20 & 1.93 & 3.90 & - \\
\hline $\mathrm{Cys}^{h}$ & - & - & - & - & - & - & - & - & - & - & - & 0.99 & - & 0.84 & - & - & - \\
\hline Val & 0.91 & 1.03 & 2.67 & 1.90 & 1.02 & 0.99 & - & - & 1.08 & - & - & - & 0.96 & 3.16 & 1.11 & 2.78 & - \\
\hline $\mathrm{Met}^{b}$ & - & - & - & - & - & - & - & - & - & - & - & - & - & - & - & - & - \\
\hline Leu & 1.06 & 1.03 & 1.07 & 2.09 & 1.10 & - & - & - & 1.97 & 1.98 & - & 2.10 & 1.02 & $3.64(4)$ & - & 1.01 & - \\
\hline Tyr & - & - & - & 0.83 & - & - & - & - & - & - & - & - & - & - & 1.00 & - & 0.99 \\
\hline Phe & - & - & - & - & 2.84 & - & - & - & 0.98 & - & 0.87 & - & 0.96 & 1.00 & 0.97 & - & - \\
\hline His & 1.00 & - & - & - & - & - & 1.10 & - & - & - & - & 1.04 & 0.96 & $1.69(2)$ & - & 0.98 & 0.89 \\
\hline $\operatorname{Trp}^{b}$ & - & 0.82 & - & 0.81 & - & - & - & - & - & - & - & - & - & - & - & - & - \\
\hline Lys & 1.12 & 0.81 & - & - & 1.10 & 1.00 & 1.11 & 1.00 & 1.02 & 1.12 & 1.14 & 1.09 & 0.98 & 0.97 & 1.11 & 1.02 & - \\
\hline Arg & 一 & - & 1.05 & 0.92 & - & - & - & - & - & - & - & - & - & - & - & - & - \\
\hline Sum & 8 & 9 & 13 & 10 & 19 & 2 & 4 & 1 & 10 & 6 & 5 & 8 & 9 & 16 & 12 & 12 & 2 \\
\hline
\end{tabular}

${ }^{b}$ Determined after performic acid oxidation. Values within parentheses are taken from sequence analysis.

Table IV. Amino Acid Differences Between $\alpha \mathrm{I} / \alpha \mathrm{II}$ Chains of Fur Seal with Those of Other Carnivora Sequences

\begin{tabular}{|c|c|c|c|c|c|c|c|c|c|c|c|c|c|c|c|c|c|c|c|c|c|c|c|c|c|c|c|c|}
\hline \multicolumn{29}{|c|}{ A. Fur seal $\alpha$ I chain } \\
\hline Pos: & 8 & 10 & 12 & 17 & 19 & 23 & 30 & 34 & 35 & 50 & 57 & 68 & 70 & 71 & 72 & 73 & 78 & 80 & 85 & 89 & 100 & 111 & 115 & 116 & 129 & 130 & 131 & Diff. \\
\hline FSI & $\mathrm{T}$ & I & $\mathrm{T}$ & L & $\mathrm{G}$ & $\mathrm{E}$ & $\mathrm{E}$ & $\mathbf{M}$ & $\mathrm{S}$ & $\mathrm{P}$ & A & $\mathbf{T}$ & V & A & $\mathbf{H}$ & $\mathbf{M}$ & K & $\mathrm{L}$ & A & $\mathrm{Y}$ & $\mathbf{L}$ & $\mathrm{C}$ & $\mathrm{A}$ & $\mathrm{Q}$ & $\mathrm{F}$ & $\mathrm{S}$ & $\mathrm{T}$ & 0 \\
\hline FSII & & $\mathrm{V}$ & & & & & & $\mathrm{T}$ & A & $\mathrm{H}$ & & & & & & & $\mathrm{G}$ & & & & & & & & & & $A$ & 6 \\
\hline HS & & $\mathrm{V}$ & A & I & & & & $\mathrm{T}$ & A & $\mathrm{H}$ & & & & & & & $\mathrm{G}$ & & & $\mathrm{H}$ & & & & $\mathrm{D}$ & & & A & 10 \\
\hline WS & & $\mathrm{V}$ & & I & & & & & A & & & & & $\mathrm{S}$ & & I & $\mathrm{G}$ & & & & & & & D & & & $A$ & 8 \\
\hline WR & & V & & & & & & & & & & & & & & I & $\mathrm{G}$ & & & & & & & & & & & 3 \\
\hline $\mathrm{PC}$ & & V & $\mathrm{S}$ & I & & & & A & & $\mathrm{H}$ & & $\mathrm{N}$ & & & & & $\mathrm{G}$ & & $\mathrm{D}$ & & & & & & & & A & 9 \\
\hline EM & & V & $\mathrm{S}$ & I & & & & A & & $\mathbf{H}$ & & $\mathrm{N}$ & & & & & $\mathrm{G}$ & $\mathbf{M}$ & $\mathrm{D}$ & & & & & & & & $\mathrm{A}$ & 10 \\
\hline $\mathrm{RA}$ & A & & A & I & & & & A & & & & L & & & $\mathrm{G}$ & $\mathrm{L}$ & $\mathrm{G}$ & & D & & & & & $\mathrm{E}$ & $\mathrm{L}$ & $T$ & $\mathrm{~S}$ & 13 \\
\hline EB & A & & A & & & & & A & & $\mathrm{H}$ & $G$ & $\mathrm{~N}$ & & & & $\mathrm{~L}$ & $G$ & & $\mathrm{D}$ & & & & & $\mathrm{E}$ & $L$ & & $\mathrm{~S}$ & 12 \\
\hline $\mathbf{L P}$ & & V & $\mathbf{S}$ & & & & & A & & & & $\mathrm{L}$ & & $\mathrm{G}$ & & $\mathrm{L}$ & $\mathrm{G}$ & & $\mathrm{D}$ & $\mathrm{H}$ & & & & E & & & $\mathrm{A}$ & 11 \\
\hline GP & & & $\mathrm{A}$ & I & & & & A & & & & $\mathrm{L}$ & & $\mathrm{G}$ & & $\mathrm{L}$ & $\mathrm{G}$ & & $\mathrm{D}$ & $\mathrm{H}$ & & $\mathrm{S}$ & & $\mathrm{E}$ & & & $\mathrm{A}$ & 12 \\
\hline PB & $S$ & & A & I & $\mathrm{S}$ & & & A & & & & & A & $\mathrm{G}$ & & $\mathrm{L}$ & $\mathrm{G}$ & & $\mathrm{D}$ & $\mathrm{H}$ & $\mathrm{F}$ & $\mathrm{S}$ & & $\mathrm{E}$ & & & A & 15 \\
\hline DD & & & $\mathbf{S}$ & & & D & $\mathrm{D}$ & $Q$ & & & & & & & & $\mathrm{~L}$ & $\mathrm{G}$ & & $\mathrm{D}$ & & & & A & $\mathrm{E}$ & & $A$ & $\mathrm{~A}$ & 11 \\
\hline $\mathrm{CO}$ & & & $\mathbf{S}$ & & & D & D & $Q$ & & & & & & & & $L$ & $\mathrm{G}$ & & $\mathrm{D}$ & & & & $\mathrm{T}$ & $\mathrm{E}$ & & $\mathrm{T}$ & A & 11 \\
\hline \multicolumn{29}{|c|}{ B. Fur seal $\alpha$ II chain } \\
\hline Pos: & 8 & 10 & 12 & 15 & 17 & 19 & 23 & 30 & 34 & 35 & 50 & 57 & 68 & 70 & 71 & 72 & 73 & 80 & 85 & 89 & 100 & 111 & 115 & 116 & 129 & 130 & 131 & Diff. \\
\hline FSII & $\mathrm{T}$ & V & $\mathrm{T}$ & $\mathrm{D}$ & $\mathrm{L}$ & $\mathrm{G}$ & $\mathrm{E}$ & $\mathrm{E}$ & $\mathrm{T}$ & A & $\mathrm{H}$ & A & $\mathbf{T}$ & $\mathrm{V}$ & A & $\mathrm{H}$ & M & $\mathrm{L}$ & A & $\mathrm{Y}$ & $\mathrm{L}$ & $\mathrm{C}$ & A & $Q$ & F & $\mathrm{S}$ & $A$ & 0 \\
\hline HS & & & A & & I & & & & & & & & & & & & & & & $\mathrm{H}$ & & & & $\mathrm{D}$ & & & & 4 \\
\hline WS & & & & $\mathrm{G}$ & I & & & & $\mathbf{M}$ & & $\mathrm{P}$ & $\mathrm{T}$ & & & $\mathrm{S}$ & & I & & & & & & & $\mathrm{D}$ & & & & 8 \\
\hline WR & & & & & & & & & $\mathbf{M}$ & $\mathrm{S}$ & $\mathrm{P}$ & & & & & & I & & & & & & & & & & $\mathrm{T}$ & 5 \\
\hline PC & & & $S$ & & I & & & & A & $\mathrm{S}$ & & & $\mathbf{N}$ & & & & & & $\mathrm{D}$ & & & & & & & & & 6 \\
\hline EM & & & $S$ & & I & & & & $\mathrm{A}$ & $\mathrm{S}$ & & & $\mathrm{N}$ & & & & & $\mathrm{M}$ & $\mathrm{D}$ & & & & & & & & & 7 \\
\hline RA & A & I & A & & I & & & & A & S & $P$ & & $\mathbf{L}$ & & & $\mathrm{G}$ & $\mathrm{L}$ & & $\mathrm{D}$ & & & & & E & $\mathrm{L}$ & $\mathbf{T}$ & S & 15 \\
\hline EB & $\mathbf{A}$ & I & A & & & & & & A & $\mathrm{S}$ & & $\mathrm{G}$ & $\mathrm{N}$ & & & & L & & $\mathrm{D}$ & & & & & E & $\mathrm{L}$ & & $S$ & 12 \\
\hline LP & & & $\mathrm{S}$ & & & & & & A & $\mathrm{S}$ & $\mathrm{P}$ & & $\mathrm{L}$ & & $\mathrm{G}$ & & $\mathrm{L}$ & & $\mathrm{D}$ & $\mathrm{H}$ & & & & $\mathrm{E}$ & & & & 10 \\
\hline GP & & I & A & & I & & & & $\mathbf{M}$ & $\mathrm{S}$ & $\mathbf{P}$ & & $\mathrm{L}$ & & $G$ & & L & & $\mathrm{D}$ & $\mathrm{H}$ & & $\mathrm{S}$ & & $\bar{E}$ & & & & 13 \\
\hline PB & $\mathbf{S}$ & I & A & & I & $S$ & & & $\mathbf{M}$ & $S$ & $P$ & & & A & $\mathrm{G}$ & & L & & $D$ & $\mathrm{H}$ & $\mathbf{F}$ & $S$ & & $\mathrm{E}$ & & & & 16 \\
\hline DD & & I & $\mathbf{S}$ & & & & D & D & $\mathrm{Q}$ & $S$ & $P$ & & & & & & $\mathrm{~L}$ & & $\mathrm{D}$ & & & & $\mathrm{T}$ & $\mathrm{E}$ & & A & & 12 \\
\hline $\mathrm{CO}$ & & I & $S$ & & & & $\mathrm{D}$ & D & $Q$ & $S$ & $\mathrm{P}$ & & & & & & $\mathrm{L}$ & & $\mathrm{D}$ & & & & $T$ & $\mathrm{E}$ & & $\mathrm{T}$ & & 12 \\
\hline
\end{tabular}

${ }^{a}$ Only differences are marked by the one-code designation of the respective amino acids. Abbreviations used for species are: FS, fur seal; HS, harbor seal; WS, Weddell seal; WR, walrus; PC, polecat; EM, mink; RA, racoon; EB, badger; LP, lesser panda; GP, giant panda; $\mathrm{PB}$, polar bear; DD, dog; and CO, coyote. 
Table V. Comparison of Fur Seal and Other Carnivora $\beta$-Chains ${ }^{a}$

\begin{tabular}{|c|c|c|c|c|c|c|c|c|c|c|c|c|c|c|c|c|c|c|c|c|c|c|c|c|c|}
\hline \multicolumn{26}{|c|}{$\beta$-chains } \\
\hline Pos: & 5 & 6 & 9 & 10 & 12 & 13 & 16 & 21 & 27 & 33 & 41 & 43 & 50 & 51 & 52 & 54 & 56 & 58 & 73 & 95 & 104 & 125 & 135 & 139 & Diff \\
\hline FS & A & $D$ & A & A & $\mathrm{T}$ & A & G & D & $\mathrm{E}$ & $\mathrm{V}$ & $\mathrm{F}$ & D & $\mathrm{S}$ & $\mathbf{P}$ & D & $\mathrm{V}$ & $\mathrm{G}$ & $P$ & D & K & $\mathrm{K}$ & $\mathrm{Q}$ & A & $\bar{N}$ & 0 \\
\hline HS & $\mathrm{G}$ & E & $\mathrm{S}$ & & & & & & & & & & & A & & I & & & & & & & & & 5 \\
\hline WS & & $\mathrm{E}$ & $\mathrm{S}$ & & & & & & & & & & & & $\mathrm{N}$ & I & $\mathrm{S}$ & & & $Q$ & & & & & 6 \\
\hline WR & & & & & & & & & & & & & & & & & & & & & & & & & 0 \\
\hline $\mathrm{PC}$ & $\mathrm{G}$ & $\mathrm{E}$ & & & & & & & $\mathrm{T}$ & & & & & & & & & & E & & & & $\mathrm{T}$ & & 5 \\
\hline EM & & $\mathrm{E}$ & & & & & & & & & & & & & & & & & E & & & & & $\mathrm{T}$ & 3 \\
\hline $\mathrm{RA}$ & & & $\mathrm{T}$ & & & $\mathrm{T}$ & & $E$ & & & & E & & A & & I & & & E & & $\mathbf{R}$ & $\mathbf{P}$ & & & 9 \\
\hline $\mathrm{EB}$ & & E & $\mathrm{S}$ & & & $\mathrm{S}$ & & & & & $\mathrm{Y}$ & & $\mathrm{T}$ & & & & & & $\mathrm{E}$ & & & & & & 6 \\
\hline LP & $\mathrm{G}$ & $\mathrm{E}$ & & & & $\mathrm{G}$ & $\mathrm{S}$ & & & & & & & & & & & & E & & & & & & 5 \\
\hline GP & $\mathrm{G}$ & $\mathrm{E}$ & & & & $G$ & $\mathrm{~S}$ & & & & & & $\mathrm{~T}$ & & & & $\mathrm{~N}$ & & $\mathrm{E}$ & & & & & & 7 \\
\hline PB & G & $\mathrm{E}$ & $\mathrm{S}$ & & & $\mathrm{G}$ & $\mathrm{G}$ & & & & & & $\mathrm{T}$ & A & & I & $\mathrm{N}$ & & & & & & & & 9 \\
\hline DD & & $\mathrm{E}$ & $\mathrm{S}$ & $\mathrm{L}$ & $\mathrm{S}$ & $\mathrm{G}$ & & & & I & & & $\mathrm{T}$ & & & & $\mathrm{S}$ & A & & & & & & & 9 \\
\hline $\mathrm{CO}$ & & $\mathrm{E}$ & S & $\mathrm{L}$ & $S$ & $\mathrm{G}$ & & & & I & & & $\mathrm{T}$ & & & & $\mathrm{S}$ & A & & & & & & & 9 \\
\hline
\end{tabular}

${ }^{a}$ For abbreviations, see Table IV.

NA

A

AB $\quad 8$

Hu $\propto$ Val Ala Gly Val Ala 20

FS $\propto I$

FS

Val- -Leu-Ser-Pro-Ala-Asp-Lys-Thr-Asn-I le-Lys-Thr-Thr-Trp-Asp-Lys-Leu-Gly-Gly-His-Ala-Gly-Glu-TyrVal-His-Leu-Thr-Ala-Asp-Glu-Lys-Ala-Ala-Val-Thr-Ala-Leu-Trp-Gly-Lys-Val- -Asn-Val-Asp-Glu-ValNA Pro Ser 10

A

A

c CD

Hu $\propto$
FS
FS
Hu
Hu

Ala 30 Met Leu
Gly-Gly-Glu-Ala-Leu-Glu-Arg-Thr-Phe-Met-Ser-Phe-Pro-Thr-Thr-Lys-Thr-Tyr-Phe-Pro-His-Phe-
Gly-Gly-G -Asp-Leu-Ala-Leu-Gly-Arg-Leu-Leu-Val-Val-Tyr-Pro-Trp-Thr-Gin-Arg-Phe-Phe-Asp-Ser-Phe-Gly-Asp-Leu-
30

- D

Hu
FS
FS
Hu

His

Asn Ser-Ser-Pro-Asp-Ala-Val-Met-Gly-Asn-Pro-Lys-Val-Lys-Ala-His-Gly-Lys-Lys-Val-Leu-Asn-Ser-Phe-Ser-AspSer-Ser-Pro-Asp-Ala-Val-Met-Gly-Asn-Pro-Lys-Val-Lys-Ala-His-Gly-Lys-Lys-Val-LeU-Asn-Ser-Phe
Ghr 60 Gly-Ala EF

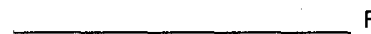
$\mathrm{F}$

Hu $\propto$ Val

Met Asn

80 His 90

FS oxI Ala-Val-Ala-His-Met-Asp-Asp-Leu-Pro-Lys-Ala-Leu-Ser-Ala-Leu-Ser-Asp-Leu-His-Ala-Tyr-Lys-Leu-Arg-ValFS Gly-Leu-Lys-Asn-Leu-Asp-Asn-Leu-Lys-Gly-Thr-Phe-Ala-Lys-Leu-Ser-Glu-LeU-H is-Cys-Asp-Lys-Leu-His-ValHu Gly-Leu-Lys-Asn-Leu-Asp-Asn-Leu-Lys-Gly-Thr-Phe-Ala-Lys-Leu-Ser-Gl
Ala-His $\mathrm{EF}$ $\mathbf{F}$ 90 FG

G $\mathrm{GH}$ H

Hu $\alpha$

FS $\alpha I$

FS $\mathrm{B}$

100

$110 \mathrm{Ala}$

Leu

Asp-Pro-Val-Asn-Phe-Lys-Leu-Leu-Ser-His-Cys-Leu-Leu-Val-Thr-Leu-Ala-Cys-His-His-Pro-Ala-Glu-Phe-ThrAsp-Pro-Glu-Asn-Phe-Lys-Leu-Leu-Gly-Asn-Val-Leu-Val-Cys-Val-Leu-Ala-His-His-Phe-Gly-Lys-Glu-Phe-ThrG 100 Arg

110 GH 120 H

HC

$\begin{array}{ll}\text { Hu } & \alpha \\ \text { FS } & \alpha I \\ \text { FS } & \beta\end{array}$

Hu $\beta$

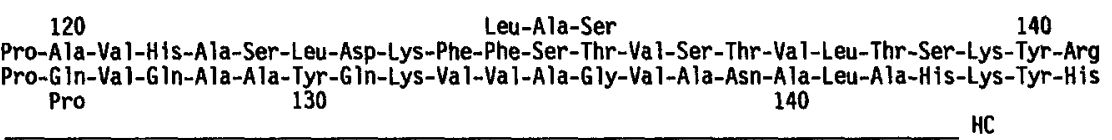

Fig. 4. Amino acids sequence of the $\alpha$ and $\beta$ chains from fur seal (FS) hemoglobins. The sequences are aligned with the chains of adult human $(\mathrm{Hu})$ hemoglobin. For the latter, only the substituted residues are given. The helices are indicated. The Hb-II differ from the Hb-I at $\alpha \mathrm{I} / \alpha \mathrm{II}: \alpha 10 \mathrm{Ile} / \mathrm{Val}, \alpha 34 \mathrm{Met} / \mathrm{Thr}, \alpha 35 \mathrm{Ser} / \mathrm{Ala}, \alpha 50$ Pro/ His, $\alpha 78 \mathrm{Lys} / \mathrm{Gly}$, and $\alpha 131 \mathrm{Thr} / \mathrm{Ala}$. 
comparisons in Tables IV and V disclose very interesting results, viz., that within marine Carnivora, an identical $\beta$-chain is found in fur seal and walrus; whereas, 3 and 5 differences are seen comparing the $\alpha \mathrm{I}-$ and $\alpha$ II-chains, respectively. This is the only identical $\beta$-chain is so far detected in any marine Carnivora. The data shows a close relationship in marine carnivora and favors the classification in a common superfamily. It is very surprising, that 6 exchanges are found within two fur seal $\alpha$-chains ( $\alpha \mathrm{I}$ and $\alpha \mathrm{II}$ ), whereas only 4 exchanges are seen when $\alpha \mathrm{II}$ from fur seal is compared with $\alpha$-chain from harbor seal. Lower numbers of exchanges are moreover found when Pinnipedia hemoglobins are compared to mink and polecat (Mustalidae) globin chains sequences. On the contrary, the difference between the polar bear (Ursidae) and pinnipeds is clearly larger. These differences suggest a relationship between Pinnipedia and Mustalidae but not especially between Pinnipedia and Ursidae. Concentrating on the Pinnipedia, there is no common residue that is found only in these four representatives (Tables IV and V). In marine Carnivora, position $\alpha 34$, which involves $\alpha 1 \beta 1$ contacts, is unique, in having Met and Thr that are not in any other representative of terrestrial Carnivora studied so far.

\section{ACKNOWLEDGMENT}

We would like to thank Mr. R. Mentele, Ms. B. Schrank, Ms. R. Gautsch, Ms. A. Muhr, and Ms. E. Wottawa for their help in amino acid analysis and sequence work. A. Ahmed is thankful to the MaxPlanck-Gessellschaft zur Forderung der Wissenschaften e.V. for the postdoctoral fellowship award.

\section{REFERENCES}

Ahmed, A., Jahan, M., Braunitzer, G., and Pechlaner, H. (1989). Z. Naturforsch. 44b, 817-824.

Ahmed, A., Jahan, M., and Braunitzer, G. (1990). Z. Naturforsch. 45c, 223-228.

Anson, M. L., and Mirsky, A. F. (1930). J. Gen. Physiol. 13, 469-476.

Alter, B. P., Goff, S. C., Efremov, G. D., Gravely, M. E., and Huisman, T. H. J. (1980). Br. J. Haematol. 44, 527-534.

Begg, G., Ruecknagel, P., Godovac-Zimmerman, J., Braunitzer, G., and Schuster, W. (1986). Biochem. Hoppe-Seyler 367, 81-82.

Braunitzer, G., Schrank, B., and Ruhfus, A. (1970). Hoppe-Seylers Z. Physiol. Chem. 351, 1569-1590.

Braunitzer, G., Schrank, B., Stangl, A., and Scheithauer, U. (1978) Hoppe-Seylers Z. Physiol. Chem. 359, 137-146.

Brimhall, B., Duerst, M., and Jones, R. T. (1977a). J. Mol. Evol. 9, 231-235.

Brimhall, B., Stangland, K., Jones, R. T., Becker, R. R., and Bailey, T. J. (1978). Hemoglobin 2, 351-370.

Clegg, J. B., Naughton, M. A., and Weatherall, D. J. (1966). J. Mol. Biol. 19, 91-108.

Edman, P., and Begg, G. (1967). Eur. J. Biochem. 1, 80-91.

Hirs, C. H. W. (1967). Methods Enzymol. 11, 218-220.

Hofman, O., Braunitzer, G., and Goeltenboth, R. (1987), Biol. Chem. Hoppe-Seyler 368, 507-511.

Hofman, O., and Braunitzer, G. (1987). Biol. Chem. Hoppe-Seyler $368,949-954$.

Hombrados, I., Ducastaing, S., Iron, A., Neuzil, E., Debuire, B., and Han, K. K. (1976). Biochim. Biophys. Acta 427, 107-118.

Hombrados, I., Neuzil, E., Debuire, B., and Han, K. K. (1978). Biochim. Biophys. Acta 535, 1-10.

Kratzin, H., Yang, C., Krusche, U., and Hilschmann, N. (1980). Hoppe Seylers $Z$. Physiol. Chem. 361, 1591-1598.

Lin, H.-X., Kleinschmidt, T., Johnson, M. L., and Braunitzer, G. (1989). Biol. Chem. Hoppe Seyler 370, 135-140.

Lin, H.-X., Kleinschmidt, T., Braunitzer, G., and Scheil, H. G. (1989). Biol. Chem. Hoppe-Seyler 370, 707-713.

Pfletschinger, J., and Braunitzer, G. (1980). Hoppe-Seyler Z. Physiol. Chem. 361, 925-931.

Runkel, D., Dresler, S. L., Brimhall, B., and Jones, R. T. (1974) Biochem. Genet. 12, 467-473.

Tagle, D. A., Miyamoto, M. M., Goodman, M., Hofmann, O., and Braunitzer, G. (1986). Naturwissenschaften 73, 512-514.

Watanabe, B., Maita, T., Matsuda, G., and Johnson, M. L. (1986) Biol. Chem. Hoppe-Seyler 367, 1251-1258.

Zimmermann, C. L., and Pisano, J. (1977). Meth. Enzymol. 57, $45-51$. 